\title{
La conversión a líquidos de diálisis peritoneal con bicarbonato puede afectar la volemia de los pacientes
}

\author{
Francisco Cirera Segura, Jesús Lucas Martín Espejo, Macarena Reina Neyra
}

Enfermeros. Unidad de Gestión Clínica Uro-Nefrológica. Hospital Universitario Virgen del Rocío. Sevilla

\section{Resumen}

El objetivo fue evaluar la repercusión del cambio de tampón utilizado en los líquidos de diálisis peritoneal, especialmente sobre los parámetros relacionados con la volemia y sobre la calidad de la diálisis.

\section{Material y métodos}

Se realizó un estudio descriptivo retrospectivo de 6 meses de duración. La muestra estuvo compuesta por 18 pacientes.

Se recogieron todas las variables de la historia clínica digital del último trimestre del año 2011 cuando los pacientes utilizaban líquidos con lactato, y a los tres meses de utilizar las soluciones con bicarbonato.

El análisis se realizó con el paquete estadístico IBM SPSS Statistics 19.0.

\section{Resultados}

La muestra la formaron 18 pacientes, el $61,1 \%$ eran hombres. La edad media fue $72,5 \pm 1,43$ años. El $55,6 \%$ estaban en diálisis peritoneal continua ambulatoria y $44,4 \%$ en automática.

Correspondencia:

Francisco Cirera Segura.

Ángel Ripoll Pastor, $n^{\circ} 4,6^{\circ} \mathrm{A}$

41006 Sevilla

Email: paco.cirera@gmail.com
Comparamos la función renal residual, y las variables implicadas en los cambios de volemia. Aunque disminuyeron la diuresis, la ultrafiltración y la función renal residual, siendo sólo significativo el último caso $(p=0.031)$ y aumentó el peso, pudo conseguirse con el aumento del número de intercambios en el $27,8 \%$ de los casos o de aumentar la concentración de glucosa también en el $27,8 \%$.

Se comprobó que el cambio de líquidos no afectó a la dosis de diálisis peritoneal ni renal. Tampoco afectó al trasporte peritoneal.

\section{Conclusión}

Tras el cambio de las soluciones a bicarbonato, nuestros pacientes han mostrado una menor ultrafiltración, diuresis y aumento de peso, que junto con el aumento en cinco casos de las concentraciones de glucosa y en otros cinco el número de intercambios, nos llevan a valorar más detenidamente los parámetros relacionados con la volemia en los pacientes que utilizan estas nuevas soluciones.

\section{PALABRAS CLAVE:}

- DIÁLISIS PERITONEAL

- LÍQUIDO DE DIÁLISIS

- BICARBONATO 


\section{Conversion to peritoneal dialysis fluids with bi- carbonate can affect patient blood volume}

\section{Abstract}

The aim was to assess the repercussion of changing the buffer used in peritoneal dialysis fluids, especially on parameters related to blood volume and on the quality of the dialysis.

\section{Material y methods}

A 6-month retrospective descriptive study was carried out. The sample was composed of 18 patients.

All the variables of the digital medical history for the last quarter of 2011 were compiled when the patients used fluids with lactate, and for the last three months when they used solutions with bicarbonate.

The analysis was carried out suing the IBM SPSS Statistics 19.0 statistical package.

\section{Results}

The sample comprised 18 patients, $61.1 \%$ of which were men. The mean age was $72.5 \pm 1.43$ years. $55.6 \%$ were on continuous ambulatory peritoneal dialysis and $44.4 \%$ on automatic dialysis.

We compared residual renal function and the variables involved in changes in blood volume. Although diuresis, ultrafiltration and residual renal function decreased, only the last of these being significant $(p=0.031)$ and weight increased, it was possible to achieve it by increasing the number of exchanges in $27.8 \%$ of cases or by increasing the glucose concentration also in $27.8 \%$.

It was found that the change of fluid did not affect the peritoneal or renal dialysis dose. Peritoneal transport was also unaffected.

\section{Conclusion}

After changing solutions to bicarbonate, our patients have shown less ultrafiltration and diuresis and an increase in weight, which together with the increase in glucose concentrations in five cases and in the number of exchanges in another five cases, lead us to evaluate more closely the parameters related to blood volume in patients who use these new solutions.

\section{KEY WORDS:}

- PERITONEAL DIALYSIS

- DIALYSIS FLUID

- BICARBONATE

\section{Introducción}

Actualmente algunos autores consideran como principal problema de la Diálisis Peritoneal (DP) a corto plazo, la infección peritoneal, a medio plazo la adecuación de la diálisis y a largo plazo la membrana peritoneal sometida cada vez a mayores volúmenes de líquido no fisiológico․ Por lo tanto, la necesidad de disponer de soluciones de diálisis que mejoren la evolución clínica de los pacientes y poder individualizar el tratamiento en base a esas soluciones, ha llevado al desarrollo de nuevas formulaciones en el líquido de diálisis².

Entre los factores descritos en la literatura que pueden afectar la biocompatibilidad de la diálisis peritoneal se incluyen el pH bajo y el tipo de buffer de las soluciones. Las soluciones de bicarbonato tienen un $\mathrm{pH}$ de 7,0 a 7,4 y muchas de las funciones celulares que se inhibían con el lactato, parecen mejorar con los líquidos peritoneales que utilizan el bicarbonato como tampón, ya que podrían proporcionar una mejor protección de la membrana peritoneal y mantener la función renal residual ${ }^{3,4}$.

En nuestra unidad, a finales del año 2011 se realizó la conversión de todos los pacientes que utilizaban líquidos con lactato a bicarbonato. Nos pareció observar una disminución de la ultrafiltración así como un aumento de las concentraciones de glucosa prescritas.

Por todo lo expuesto, nos planteamos como objetivo evaluar la repercusión del cambio de tampón utilizado en los líquidos de diálisis peritoneal, especialmente sobre los parámetros relacionados con la volemia como la diuresis, peso y la aparición de edemas así como sobre la calidad de la diálisis. 


\section{Material y método}

Se realizó un estudio descriptivo retrospectivo de 6 meses de duración, cuya población fueron todos los pacientes activos en nuestro programa de diálisis peritoneal con función renal residual, que pasaron de utilizar líquidos con lactato a bicarbonato. El cambio se produjo el 20 de diciembre del año 2011. La muestra estuvo compuesta por 18 pacientes; 11 hombres y 7 mujeres.

Se recogieron las siguientes variables de la historia clínica digital correspondientes al último trimestre del año 2011 cuando los pacientes aún utilizaban líquidos con lactato, y a los tres meses de utilizar las nuevas soluciones con bicarbonato:

- Demográficas: edad, género, patología de base. ${ }^{5}$

- Datos relacionados con la volemia: peso, presencia de edemas, diuresis residual, función renal residual y necesidad de aumentar el número de intercambios o concentración de glucosa de los mismos.

- Datos relacionados con la técnica de diálisis: modalidad de tratamiento, dosis de diálisis (Kt/V) peritoneal y renal, test de equilibrio peritoneal.

Para realizar la estadística descriptiva de las variables del estudio se usaron frecuencias absolutas y relativas en el caso de las variables cualitativas. Las cuantitativas según si su distribución fuera normal o no, se resumieron mediante la media y la desviación estándar o la mediana y el rango intercuartílico respectivamente. Realizamos la comprobación de los grupos del estudio mediante el test de McNemar o el test de Wilcoxon, y para la comparación de variables cuantitativas la $T$ de Student para datos apareados.

El análisis se realizó con el paquete estadístico IBM SPSS Statistics 19.0, estableciendo el nivel de significación en $p<0,05$.

\section{Resultados}

La muestra la formaron total 18 pacientes, donde el $61,1 \%$ eran hombres $(n=11)$. La edad media fue 72,5 $\pm 11,43$ años. El 55,6\% ( $n=10)$ estaban en diálisis peritoneal continua ambulatoria (D.P.C.A.) y $44,4 \%(n=8)$ en diálisis peritoneal automática (D.P.A). La etiología de la Enfermedad Renal Crónica se muestra en la Tabla
1. Los pacientes Ilevaban al inicio del estudio una media de $26,42 \pm 16,42$ meses (rango entre 10-64 meses) en DP.

\begin{tabular}{|c|c|}
\hline & Porcentaje válido (Frecuencia) \\
\hline No Filiada & $61,1 \%(n=11)$ \\
\hline Enfermedades Vasculares & $16,7 \%(n=3)$ \\
\hline Nefropatía Diabética & $11,1 \%(n=2)$ \\
\hline Glomerulonefritis & $5,6 \%(n=1)$ \\
\hline Enfermedades Sistémicas & $5,6 \%(n=1)$ \\
\hline
\end{tabular}

TABLA 1. Etiología de la Enfermedad Renal Crónica*.

*Códigos EDTA 1994-1995 y su agrupación, extraídos del Informe 2006. Módulo Básico. Subsistema de Insuficiencia Renal Crónica. Servicio Andaluz de Salud. Junta de Andalucía.

En primer lugar comparamos la función renal residual de los pacientes, así como las variables implicadas en los cambios de volemia como el peso, la diuresis, la ultrafiltración y la presencia de edemas. Los resultados se recogen en la Tabla 2.

\begin{tabular}{|c|c|c|c|}
\hline $\begin{array}{c}\text { Función Renal } \\
\text { Residual }\end{array}$ & $\mathbf{4 , 2 3}(2,2-5,17)$ & $\mathbf{3 , 2}(1,9-4,81)$ & $\mathbf{0 , 0 3 1}$ \\
$\begin{array}{c}\text { Diuresis (24 } \\
\text { horas) }^{\mathbf{1}}\end{array}$ & $\mathbf{9 1 2 , 5}(600-1325)$ & $\mathbf{8 2 5}(300-1087,5)$ & 0,1 \\
\hline $\begin{array}{c}\text { Ultrafiltración } \\
\text { 2 }\end{array}$ & $\mathbf{9 8 9 , 2 7} \pm 378,73$ & $\mathbf{8 9 8 , 5 5} \pm 330,63$ & 0,35 \\
\hline $\begin{array}{c}\text { Peso }^{2} \\
\text { Presencia de }^{\text {edemas }}\end{array}$ & $\mathbf{7 8 , 1 8} \pm 15,35$ & $\mathbf{7 9 , 5 5} \pm 16,35$ & 0,23 \\
\hline
\end{tabular}

TABLA 2. Función Renal Residual y variables relacionadas con la volemia.

${ }^{1}$ Prueba de Wilcoxon. ${ }^{2} \mathrm{~T}$ de Studen para muestras apareadas. ${ }^{3} \mathrm{Chi}$ cuadrado

Aunque disminuyeron la diuresis, la ultrafiltración y la función renal residual (sólo significativamente en el último caso) y aumentó el peso, estudiamos si esto pudo conseguirse debido al aumento del numero de intercambios, lo que ocurrió en el $27,8 \%$ de los casos $(n=5)$, o de aumentar la concentración de glucosa de los mismo, también ocurriendo en el mismo porcentaje, $27,8 \%$ $(n=5)$, existiendo 3 casos en los que hubo que aumentar al mismo tiempo la concentración de glucosa y el número de intercambios. En ningún caso los pacientes tomaban diuréticos.

De igual forma se comprobó si el cambio de líquidos afectó a la dosis de diálisis ( $\mathrm{Kt} / \mathrm{V}$ ) peritoneal y renal (Tabla 3). 


\begin{tabular}{c|c|c|c|}
\hline Kt/V peritoneal. & $\mathbf{4 , 2 3}(2,2-5,17)$ & $\mathbf{3 , 2}(1,9-4,81)$ & 0,15 \\
\hline Kt/V renal. & $\mathbf{0 , 7 5}(0,45-0,99)$ & $\mathbf{0 , 6 5}(0,34-0,86)$ & 0,74
\end{tabular}

TABLA 3. Dosis de diálisis administrada.

${ }^{1}$ Prueba de Wilcoxon.

El cambio de líquidos de diálisis peritoneal tampoco afectó al trasporte peritoneal $(p=0,49)$, aunque aumentaron los pacientes clasificados como altos trasportadores del $11,1 \%(n=2)$ al $27,8 \%(n=5)$, permaneciendo igual los medio-altos y bajando los medios-bajo de $33,3 \%(n=6)$ a $16,7 \%(n=3)$.

\section{Discusión}

Las nuevas soluciones de diálisis peritoneal han tenido interés en los últimos años, sobretodo si tenemos en cuenta el número de publicaciones. Los aspectos más destacados se refieren en conjunto a los posibles beneficios clínicos conseguidos con el uso de las mismas, a veces contradictorios.

En la bibliografía no hay acuerdo acerca de si la ultrafiltración es inferior con las soluciones biocompatibles: unos trabajos no encuentran diferencias significativas entre las dos soluciones, mientras otros observan una menor ultrafiltración con las soluciones nuevas ${ }^{6,7,8,9}$. En nuestra muestra, no encontramos diferencias significativas aunque si $100 \mathrm{ml}$ menor con las nuevas soluciones. Además en cinco pacientes hubo que aumentar el número de intercambios y en otros cinco la concentración de la glucosa y a pesar de esto, se encontró una disminución de la diuresis, y un aumento del peso respecto a los resultados obtenidos con el lactato. Esto ha supuesto aumentar la exposición del peritoneo a mayores cargas de glucosa y a aumentar el riesgo de infecciones peritoneales al tener que realizar un mayor número de conexiones.

Otra de las ventajas que aportan las soluciones con bicarbonato es la conservación de la función renal residual, que en DP ha demostrado influir en la mayor supervivencia de los pacientes, sin embargo este punto también está sujeto a controversias en la literatura ya que Montenegro y col, comunican que la función renal residual se mantiene durante más tiempo cuando se usan soluciones con bicarbonato, ${ }^{10}$ mientras otros autores comunican un descenso por igual en los pacientes que usan bicarbonato/lactato respecto a los que utilizan solo lactato $^{11}$.
En nuestro estudio, hemos analizado esta variable ya que es una de las principales ventajas de los líquidos con bicarbonato. Debido a las limitaciones de este trabajo y a su diseño y a pesar de haber observado un descenso de la función renal residual, puede que dicho descenso no sea atribuible a estas soluciones, y se deba más bien al tiempo en la técnica. De igual forma tampoco pueden deberse a cambios en el tratamiento con diuréticos ya que por protocolo de nuestro centro, los diuréticos son suspendidos desde el inicio de la DP.

A pesar del tiempo que ha transcurrido desde que se inició el uso de las soluciones con bicarbonato, hay muchos aspectos que en la práctica diaria y en las publicaciones aún no quedan suficientemente demostradas, por lo que debemos seguir investigando sus efectos en el tiempo.

Por otro lado, individualizar el tratamiento supone que aunque es necesario disponer de los últimos avances no obligatoriamente pueden beneficiar a todos los pacientes y aunque sí reconocemos otras ventajas de las soluciones con bicarbonato como una mejor corrección de la acidosis, en algunos casos sería más interesante mantener este mayor poder de ultrafiltración, más en aquellos pacientes que hayan perdido la función renal residual.

Como conclusión se observa que tras el cambio de las soluciones con lactato a bicarbonato, nuestros pacientes han mostrado una menor ultrafiltración, diuresis y aumento de peso, sin llegar a ser significativa, que junto con el aumento en cinco casos de las concentraciones de glucosa y en otros cinco el numero de intercambios, indica la necesidad de valorar más detenidamente los parámetros relacionados con la volemia en los pacientes que utilizan estas nuevas soluciones.
Recibido: 10 Abril 2013

Revisado: 20 Abril 2013

Modificado: 28 Abril 2013

Aceptado: 2 Mayo 2013 


\section{Bibliografía}

1. Gallar Ruiz, P. Resultados a medio y largo plazo en DPA. En Coronel F. (Ed.) Diálisis Peritoneal a largo plazo. Editorial Médica JIMS. Barcelona. 1998. 37-42.

2. Coronel F. Contribución de las nuevas soluciones peritoneales a la evolución del paciente en diálisis peritoneal. Rev Soc Esp Enferm Nefrol. 1999; 6:27-30.

3. Coronel F, Perez Flores I. Factores relacionados con la pérdida de función renal residual en diálisis peritoneal. Nefrología. 2008; (Supl. 6):39-44.

4. Bajo MA, del Peso, G Sanchez Villanueva, MJ et al. Nuevas soluciones de diálisis peritoneal y sus combinaciones. Nefrología 2008; (Supl 6):59-66.

5. Códigos EDTA 1994-1995 y su agrupación, extraídos del Informe 2006. Módulo Básico. Subsistema de Insuficiencia Renal Crónica. Servicio Andaluz de Salud. Junta de Andalucía.

6. Montenegro, J. Soluciones de Dialisis Peritoenal. Nefrología 2008; (Supl. 5):59-65.
7. Pajek J, Kveder R, Bren A, Gucek A, Bucar M, Skoberne $A$, et al. Short-term effects of bicarbonate/lactate buffered and conventional lactatebuffered dialysis solutions on peritoneal ultrafiltration: a comparative crossover study. Nephrol Dial Transplant 2009; 24:1617-25.

8. S. L. Fan, T. Pile, S. Punzalan, M. J. Raftery, M. M. Yaqoob. Randomized controlled study of biocompatible peritoneal dialysis solutions: effect on residual renal function. Kidney Int 2008; 73: 200-6.

9. Williams JD, Topley N, Craig KJ, et al. The EuroBalance Trial: The effect of a new biocompatible peritoneal dialisys fluid (balance) on the peritoneal membrane. Kydney Int 2004; 66:408-418.

10. Montenegro J, Saracho R, Martínez I et al. Longterm experience with pure bicarbonate peritoneal dialysis solutions. Perit Dial In 2006; 26:89-94.

11. Otte K, Gonzalez MT, Bajo MA et al. Clinical experience with a new bicarbonate/lactate peritoneal dialysis solution. Perit Dial In 2003; 23:138-145. 